\title{
Epidemiology of Measles in the Philippines
}

\author{
Michelle C. Ylade \\ Institute of Child Health and Human Development, National Institutes of Health, University of the Philippines Manila
}

\begin{abstract}
Background. Measles cases have decreased ever since vaccination has been introduced through the DOH Expanded Program on Immunization (DOH EPI) in the Philippines. Elimination is the current goal. However, despite high vaccine coverage, a nationwide outbreak occurred in 2013.
\end{abstract}

Objectives. This study aimed to present measles incidence rates before and after the introduction of the measles vaccine, describe the changing epidemiology of measles, and assess the effectiveness of measles vaccination and baseline measles reporting.

Methods. National data from 1960 to 2014 were used to determine incidence of measles and describe its epidemiology. Vaccine coverage and data on measles surveillance were used to estimate vaccine effectiveness by birth cohort and age group.

Results. Reported measles incidence decreased after the introduction of measles vaccine compared to prevaccination levels (pre-vaccination: 36 per 100,000 to 73 per 100,000; 2008-2014 confirmed measles incidence: 1.47 to 23.76 per 100,000 , suspected measles -5.7 to 58.08 per 100,000 ). A shift in the age distribution of cases was noted during the outbreak. Vaccine effectiveness by birth cohort ranged from $85-96 \%$ while the lowest VE estimate by age group was seen among those aged $26-30$ years.

Conclusion. Measles vaccine coverage should target wider age groups. Measles surveillance should be improved to help in evidence-based decisions for a more effective plan towards measles elimination.

Key Words: epidemiology, measles, Philippines

\section{INTRODUCTION}

Measles remains a major cause of death among young children even though a safe and cost-effective vaccine has been available for many years. ${ }^{1}$ Measles is a highly contagious seasonal disease that affected nearly everyone prior to the introduction of measles vaccine. ${ }^{2}$ At the time when the vaccine was not available, it was estimated that 2.6 million deaths per year occurred due to measles. Currently, deaths due to measles has decreased to approximately 134,200 globally. ${ }^{1}$ Measles vaccination resulted in a $75 \%$ reduction in measles deaths from 2000 to 2013 worldwide and active immunization with measles vaccine is considered the best means of preventing the disease with elimination as a current goal. ${ }^{1,3}$ Measles elimination has been defined as the absence

Corresponding author: Michelle C. Ylade, MD, MSc Institute of Child Health and Human Development National Institutes of Health, University of the Philippines Manila

Room 112 NIH Building, 623 Pedro Gil St. Ermita, Manila 1000, Philippines

Telephone: +6325264349

Email: mylademd@yahoo.com of endemic measles transmission in a defined geographical area for at least 12 months in the presence of a surveillance system that has been verified to be performing well. The World Health Organization (WHO) Global Vaccine Action Plan (GVAP) established the target of measles and rubella elimination in at least five WHO Regions by 2020.4,5 Measles elimination has been achieved in the WHO Region of the 
Americas since 2002, while the rest of WHO Regions have set the following target years for measles elimination: 2012 in the Western Pacific Region (WPR), 2015 in the European and Eastern Mediterranean Regions, and 2020 in the African and South-East Asia Regions. ${ }^{6}$

With the establishment of the Philippine Department of Health's Expanded Program on Immunization (EPI) in 1976, one dose of measles vaccine given at 9 months of age has been included in the program, then later on introduced nationwide in $1983 .{ }^{7,8}$ Measles, together with poliomyelitis and neonatal tetanus, is a vaccine-preventable disease (VPD) targeted for elimination. In 1998, the Philippines adopted a ten-year plan to eliminate measles. A catch-up mass immunization was conducted in 1998 with $96 \%$ reported coverage decreasing cases and deaths nationwide except in the NCR. ${ }^{7,9} \mathrm{~A}$ followup measles vaccination campaign was conducted in 2004 with $94 \%$ coverage that decreased reported cases and deaths by $96 \%$ and 99\%, respectively. ${ }^{9}$ In 2009, MCV2 was introduced into the EPI program and national coverage has been achieved since 2010. The first dose (MCV1) is a monovalent vaccine given to children 9 to 11 months old, while MMR is given as MCV2 to children aged 12 to 15 months old. Follow-up supplemental immunization activities (SIA) were conducted in 2007 (for children aged 9 months to 48 months) and in 2011 (for children aged 9 months to 9 years old) with a reported $95 \%$ and $84 \%$ coverage, respectively. ${ }^{8}$

Case-based measles surveillance commenced in 1982. ${ }^{9}$ The national measles reference laboratory in the Philippines at the Research Institute of Tropical Medicine (RITM), has been conducting serologic testing for measles since 1999 . Table 1 highlights the important events on measles control in the Philippines.

Annual reported measles cases varied in relation to SIAs, declining after SIAs have been conducted and then increasing in subsequent years. A study by Takashima et al. presented the number of reported cases and estimated percentage of MCV1, MCV2, and the timing of supplementary immunization activities in the Philippines from 1998-2014. ${ }^{8}$ From 1998, the number of measles cases rose until 2003, decreasing thereafter until reaching its lowest number in 2006. After the SIA campaign in 2007, the number of cases started to rise again. Another SIA was conducted in 2011 that decreased the number of cases to nearly 1,000 cases in 2012. However, measles cases started to increase again in 2013 due to a nationwide measles outbreak that started in Regions III, IVA, VI, and CAR. Outbreak response immunization activities for children 6-59 months were conducted in 2014 and a nationwide SIA targeting children aged 9-59 months was conducted in September 2014. During the outbreak, 37\% of reported measles cases involved individuals aged $\geq 15$ years. ${ }^{8}$

The decrease in number of reported cases for several years followed by a resurgent measles outbreak can be considered as a "post-honeymoon period" outbreak. McLean and Anderson described the "honeymoon period" as the period of low incidence after the introduction of control generated during the shift from pre-vaccination to the post-vaccination era, during which, a change in the age distribution of susceptible population occurs. ${ }^{10}$ This has been observed, for example, in "post-honeymoon period" outbreaks in Burundi and Mongolia where a shift in the age distribution of measles cases have been observed. ${ }^{11-13}$ In Burundi, they noted that $44 \%$ of measles cases were aged $>24$ months while $18 \%$ were aged $<9$ months. They also noted that school-aged children were potential sources of infection in the households especially to their younger siblings. An investigation of the outbreak revealed that measles vaccine coverage at 1 year of age improved from 0\% in 1980 to 55\% in $1988 .{ }^{11}$

In Mongolia, an increase in measles cases was initially detected in 2001 through routine surveillance. Age-specific incidence in 2001 revealed a peak in children $<1$ year of age and another peak in young adults between 15-24 years of age. The age distribution seen among cases in the outbreak was thought to be a result of the vaccination program. Children aged 2 to 14 years as of 2001 have been immunized 3 or 4 times from 1994 to 2000, hence were likely protected. They also noted that measles outbreaks in high schools, colleges, and army units coincide with entry of new students and soldier recruits. ${ }^{13}$

A successful vaccination program requires high coverage with high quality vaccine. ${ }^{14}$ Monitoring of measles vaccine effectiveness is an important measure of quality control in immunization programs to maintain confidence in the program especially in the face of continuing outbreaks. ${ }^{15}$ Vaccine effectiveness (VE) is defined as the reduction in risk among vaccinees compared to similarly exposed nonvaccinees. Vaccine effectiveness is affected by how well target groups in the population were immunized. These may reflect difficulties in maintaining proper storage conditions of a vaccine and access to health care. ${ }^{16}$ When $\mathrm{VE}$ is found to be lower than expected, detailed investigations should be carried out to determine the causes and take corrective action. ${ }^{17}$ Doshi et al. estimated measles VE on children aged 12 to 59 months in the Democratic Republic of Congo and found improper staff training and unreliable cold chain led to loss of vaccine potency and consequently decreased VE estimates. ${ }^{15}$

\section{Significance}

The outbreak that occurred in the Philippines in 2013 presents an opportunity to assess measles vaccine effectiveness, and efficiency of measles notification in the country.

\section{OBJECTIVES}

This paper determined the measles incidence rates before and after introduction of measles vaccination and describes the changing epidemiology of measles in the Philippines. Efficiency of measles vaccination was assessed by determining vaccine effectiveness by age group and birth cohort. In addition, the adequacy of measles notification in the Philippines during the pre-vaccination period was also evaluated. 
Table 1. Timeline of events on measles control in the Philippines

\begin{tabular}{|c|c|c|c|}
\hline Date & Vaccination activities & $\begin{array}{l}\text { Surveillance and } \\
\text { laboratory testing }\end{array}$ & Changes in measles notification \\
\hline 1982 & $\begin{array}{l}\text { Introduction of measles vaccine: } \\
\text { - given to 9-month-old children as a monovalent } \\
\text { measles vaccine }\end{array}$ & $\begin{array}{l}\text { Start of case-based } \\
\text { surveillance for measles }\end{array}$ & \\
\hline 1983 & Nationwide implementation of measles vaccine & & \\
\hline 1998 & $\begin{array}{l}\text { Catch-up nationwide immunization: } \\
\text { - targeted children aged } 9 \text { months-14 years old } \\
\text { - given as a monovalent measles vaccine } \\
\text { - reported coverage was } 96 \% \\
\end{array}$ & & \\
\hline 1999 & & $\begin{array}{l}\text { Start of measles } \\
\text { serologic testing at } \\
\text { RITM }\end{array}$ & \\
\hline 2004 & $\begin{array}{l}\text { Nationwide follow-up supplemental immunization } \\
\text { activity (SIA): } \\
\text { - targeted children aged } 9 \text { months- } 8 \text { years old } \\
\text { - given as a monovalent measles vaccine } \\
\text { - reported coverage was } 94 \%\end{array}$ & & \\
\hline 2007 & $\begin{array}{l}\text { Nationwide SIA: } \\
\text { - targeted children aged } 9 \text { months- } 48 \text { months } \\
\text { - given as a monovalent measles vaccine } \\
\text { - reported coverage was } 95 \%\end{array}$ & & $\begin{array}{l}\text { PIDSR started and vaccine preventable } \\
\text { diseases were included in PIDSR reporting }\end{array}$ \\
\hline 2008 & & & $\begin{array}{l}\text { Start of classifying measles as confirmed and } \\
\text { suspected cases } \\
\text { Confirmed cases: laboratory confirmed, } \\
\text { epidemiologically linked and clinically } \\
\text { confirmed measles cases }\end{array}$ \\
\hline 2009 & $\begin{array}{l}\text { Introduction of second dose of a measles containing } \\
\text { vaccine (MCV2) for children aged } 12 \text { months old }\end{array}$ & & \\
\hline 2010 & $\begin{array}{l}\text { Nationwide implementation of MCV2: } \\
\text { - target was to give MCV1 to children aged 9-11 } \\
\text { months with a monovalent measles vaccine and } \\
\text { MCV2 to children aged 12-15 months as MMR } \\
\text { (measles, mumps, rubella) }\end{array}$ & $\begin{array}{l}\text { RITM started viral } \\
\text { isolation of measles }\end{array}$ & \\
\hline 2011 & $\begin{array}{l}\text { Nationwide SIA: } \\
\text { - targeted children aged } 9 \text { months-9 years old } \\
\text { - given as MR (measles, rubella) vaccine } \\
\text { - reported coverage was } 84 \%\end{array}$ & & \\
\hline 2013 & & & $\begin{array}{l}\text { Adapted a new case classification } \\
\text { Confirmed cases: laboratory confirmed and } \\
\text { epidemiologically linked cases } \\
\text { Renaming of clinically confirmed measles as } \\
\text { clinically compatible }\end{array}$ \\
\hline $\begin{array}{l}\text { January- } \\
\text { February } \\
2014\end{array}$ & $\begin{array}{l}\text { SIA conducted in Regions 4A, 3, NCR: } \\
\text { - targeted children aged 6-59 months } \\
\text { - given as a monovalent measles vaccine } \\
\text { - no reported coverage }\end{array}$ & & \\
\hline $\begin{array}{l}\text { September } \\
2014\end{array}$ & $\begin{array}{l}\text { Nationwide SIA: } \\
\text { - targeted children aged } 9-59 \text { months } \\
\text { - given as MR vaccine } \\
\text { - reported coverage was } 91 \%\end{array}$ & & \\
\hline
\end{tabular}

\section{MATERIALS AND METHODS}

\section{Number of births, notifications and measles incidence rates}

In the 1960s, reports on notifiable diseases were included in the Philippines Vital and Health Statistics Annual Report, which became known as the Philippine Health Statistics (PHS). The PHS was annually published by the $\mathrm{DOH}$ and contained a summary of the country's statistics on natality, morbidity, notifiable diseases, and mortality. In 1987, the
DOH also started publishing the Field Health Service Information System (FHSIS) report, an annual report that gives information on the nationwide delivery of different public health programs. This includes the Expanded Program on Immunization (EPI) report and data on notifiable diseases such as measles. These publications can be downloaded from the $\mathrm{DOH}$ website. Data points were entered manually by the author to Excel spreadsheets for analysis.

To describe notifications and measles incidence rates in the Philippines, data on number of reported measles cases and 
incidence rates from 1960-2007 were collected. Notifications refer to the number of measles cases reported in a year. Measles incidence rates reported in the PHS were crude rates computed as the number of reported measles cases in a year divided by the total population of the same year multiplied by 100,000 . Despite several years with no available report, data on measles were still obtained because each annual PHS report provided a 10-year review of reported morbidity and incidence rate of notifiable diseases. Beyond 2009, PHS reports were no longer available and thus Philippine Integrated Disease Surveillance and Response (PIDSR) data were used. With this, PIDSR data set was used as the numerator to estimate measles incidence from 2008 to 2013. For consistency with previous reports, measles incidence rates from 2008 to 2013 have been estimated in the same manner as crude incidence rates reported prior to 2008. To obtain the incidence rate for 2014, the Epidemiology Bureau provided the number of suspected measles cases from January 2014 to 20 December 2014 and the number of confirmed measles cases for the whole year.

The estimated population for 2010 was obtained from the Philippine Statistics Authority and subsequent population estimates from 2011 until 2014 have been calculated based on an annual $1.73 \%$ population growth rate. The number of reported live births from 1960-2009 was obtained from PHS. The number of live births were derived from the estimated population using a crude birth rate of 19 per 1000 mid-year population for the years 2010 to $2014 .^{18}$

\section{Measles surveillance and vaccine coverage}

Before the establishment of the PIDSR system, data on notifiable diseases were based on information submitted by health personnel of different rural health units (RHU) and city or provincial health offices. The Law on Reporting of Notifiable Diseases (Republic Act 3573) mandates the immediate reporting of any notifiable disease, including measles, to the nearest health officer. Health officers must submit weekly morbidity reports to the $\mathrm{EB}$ where data were compiled and analyzed. Although the law provides for compulsory reporting of notifiable diseases, there were still deficiencies in reporting. ${ }^{19}$

The PIDSR has been implemented since 2007 and uses standard case definitions for surveillance. Suspected cases were immediately reported to the Disease Reporting Unit, Provincial or Regional Epidemiologic and Surveillance Unit, and the EB within 24 hours of detection. An initial report should be sent, followed by a case-based reporting form using a standard PIDSR case investigation form. This form should be completed by a trained health staff. Under the PIDSR, all reported measles cases should be investigated and blood specimen should be collected from suspected cases and sent to RITM for measles-specific IgM antibody testing. ${ }^{20} \mathrm{~A}$ summary of changes in measles reporting in the Philippines is also shown in Table 1. While the PIDSR Manual of Procedures specifies that notifications should be checked for completeness and consistency of data entries, it does not indicate how vaccination status of notified cases should be ascertained.

Measles vaccine coverage was obtained from the EPI department of the Department of Health. Available national vaccine coverage data for MCV1 were for 1987-1995 and 2001-2012 and for MCV2 from 2010-2012. Vaccine coverage for the years 1996-2000 were not available. Measles surveillance data were obtained with permission from EB.

\section{Vaccine effectiveness}

Vaccine effectiveness (VE) was estimated by applying the screening method to data from the 2013 to 20 December 2014. The result was multiplied by 100 to give the estimated vaccine effectiveness in percent: ${ }^{21}$

$$
V E=1-\frac{P C V}{1-P C V} \times \frac{1-P P V}{P P V}
$$

To estimate vaccine effectiveness, confirmed measles cases with vaccination status (receipt of any $\mathrm{MCV}$ ) reported during 2013 to 20 Dec 2014, measles case-based surveillance and eligible to receive MCV1 in 1987-1995 and 2001-2012 have been selected. A confirmed measles case was defined as either laboratory-confirmed or epidemiologically-linked measles case.

The available vaccine coverage from EPI included only the years 1987 to 1995 and 2001 to 2012. It was assumed that children born 01 April 1986 to 31 March 1987 would be eligible to receive MCV1 from January to December 1987 since they would have turned 9 months in 1987. Based on the birth dates provided in the case-based surveillance data, the number of cases eligible for measles vaccination between 1987 to 1995 and 2001 to 2012 were determined. These suspected cases were then classified according to case classification and reported vaccination status (receipt of any MCV). Hence, only those eligible for measles vaccination on these years and classified as a confirmed case with reported vaccination status were included in vaccine effectiveness analysis.

Following the assumption on date of birth and year of measles vaccine eligibility, vaccine effectiveness was estimated according to birth cohort. The number of confirmed cases and confirmed cases with vaccine were obtained based on the year when they were born. The number of confirmed cases who reportedly received vaccination was divided by the number of confirmed cases to obtain the proportion of cases vaccinated (PCV) within a birth cohort. The corresponding reported measles vaccine coverage represents the proportion of the population vaccinated (PPV).

To obtain vaccine effectiveness by age group, the number of confirmed cases and confirmed cases with vaccine were stratified according to age group. The proportion of cases vaccinated $(\mathrm{PCV})$ were estimated within a particular age group. The proportion of population vaccinated (PPV) for each age group was obtained by computing a pooled vaccine coverage that applied to that particular age group. Please refer to Table 2 for the reported EPI vaccine coverage. 
Epidemiology of measles in the Philippines

Table 2. EPI measles vaccine coverage from 1987 to 1995 , and from 2001 to 2012, Philippines

\begin{tabular}{cccccc} 
Year & MCV1 (\%) & Year & MCV1 (\%) & Year & MCV1 (\%) \\
\hline 1987 & 67 & 2001 & 81 & 2010 & 85 \\
1988 & 77 & 2002 & 82 & 2011 & 83 \\
1989 & 83 & 2003 & 87 & 2012 & 86 \\
1990 & 85 & 2004 & 92 & & \\
1991 & 88 & 2005 & 92 & & \\
1992 & 90 & 2006 & 92 & & \\
1993 & 88 & 2007 & 92 & & \\
1994 & 87 & 2008 & 92 & & \\
1995 & 83 & 2009 & 89 & & \\
\hline
\end{tabular}

\section{RESULTS}

Measles incidence, reported births, and notifications in the Philippines

Before the introduction of measles vaccination, reported measles incidence rates varied from 36 per 100,000 to 73 per 100,000 (Figure 1). These incidence rates refer to

the number of reported measles cases divided by the total population during the years 1960 to 2014 . The solid gray line corresponds to suspected measles incidence rates while the darker line corresponds to confirmed measles incidence rates. In 2008, classification of reported cases modified how measles cases are reported. From 2008 to 2014, suspected measles incidence rates ranged from 5.7 to 58.08 per 100,000 while confirmed measles incidence rates ranged from 1.47 to 23.76 per 100,000 .

After the introduction of measles vaccination to 9-month-old children in 1983, a sharp increase in reported incidence was noted, rising to a peak until 1987 (incidence at 141 per 100,000). After the peak, reported measles incidence rate steadily decreased until 1991 . Despite observing a spike in measles reported incidence rate to 127 per 100,000 in 1993 , incidence gradually decreased to 2.9 per 100,000 in 2009. The second dose of measles vaccine, given as MMR, was introduced in 2010. Reported measles incidence slightly decreased after the introduction of the second dose until a sharp rise was seen from 2013 to 2014.

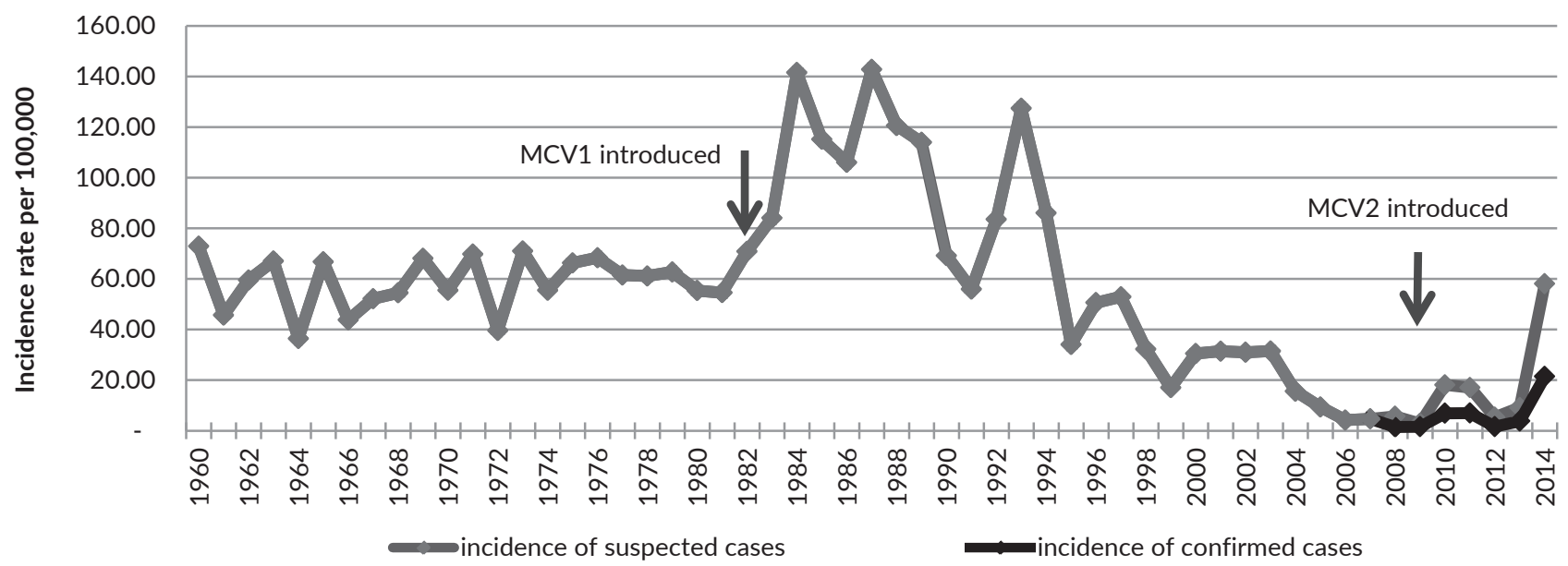

Figure 1. Suspected measles incidence rate per 100,000 from 1960 to 2014 and confirmed measles incidence rate per 100,000, 2008-2014, Philippines.

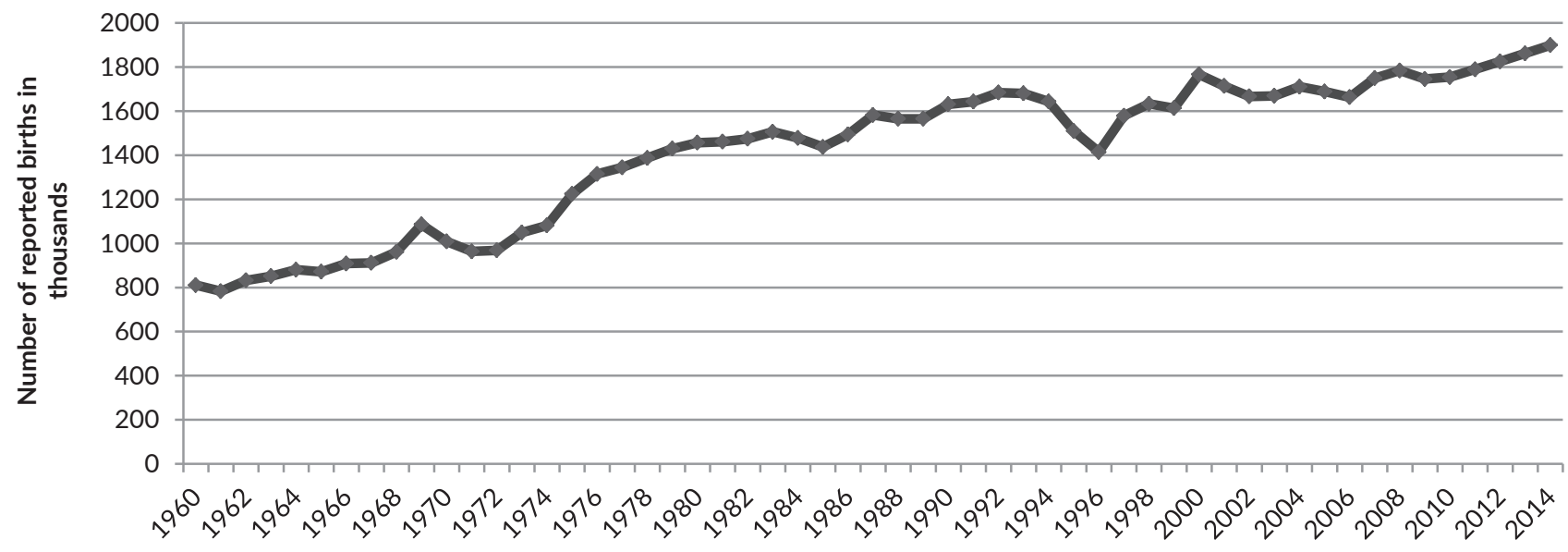

Figure 2. Number of reported births (in thousands), from 1960 to 2014, Philippines. 


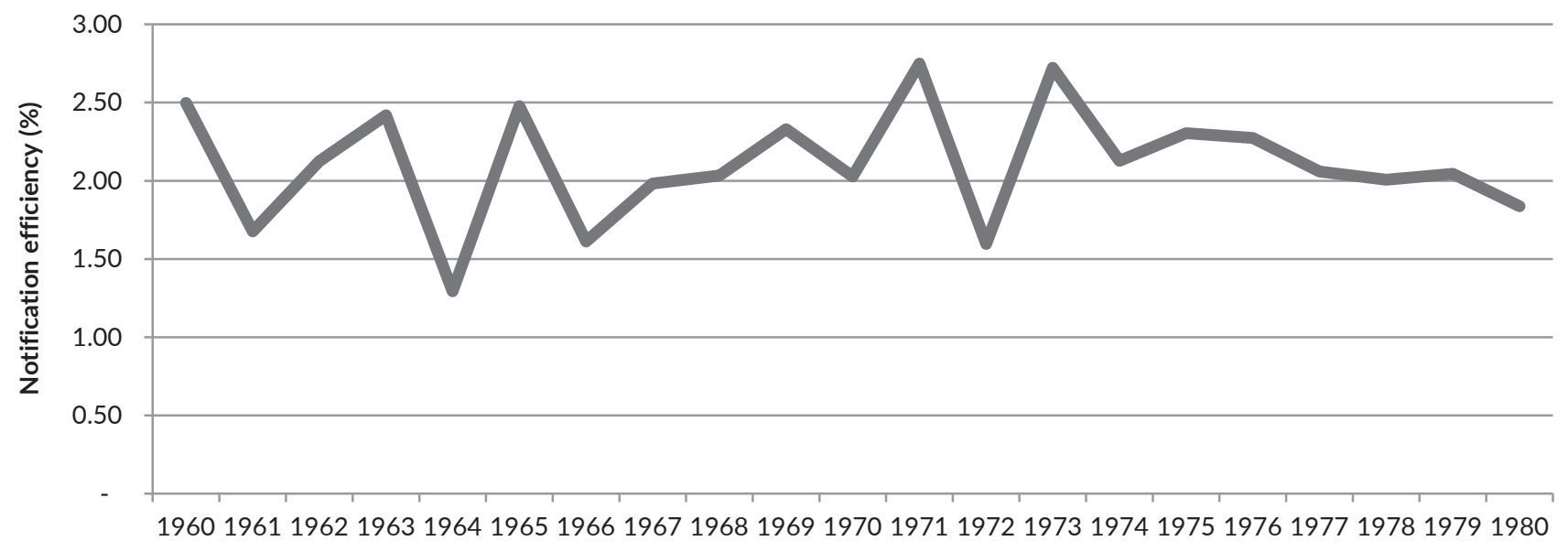

Figure 3. Estimated notification efficiency for reported measles cases from 1960 to 1980, Philippines.

In 1960, the number of live births was estimated at 810,904 increasing to $1,899,040$ in 2014 (Figure 2). Prior to the introduction of MCV1, suspected measles incidence rate and reported notifications did not follow the trend of an increase in live births until 1983 where measles notifications and suspected incidence rates suddenly increased. Given that everyone in a community ultimately contracted measles prior to the introduction of vaccination, the average yearly incidence of measles in an unvaccinated population should approximate the annual number of births. ${ }^{22}$ Notification efficiency was defined as the proportion of clinical cases of a reported notifiable disease. ${ }^{22}$ To estimate the notification efficiency before the introduction of MCV1, the number of notifications in 1960 was divided by the reported live births. Measles notification efficiency in 1960 was 2.5\%, 2.0\% in 1970 and $1.8 \%$ in 1980 . Figure 3 shows the estimated notification efficiency for reported measles cases from 1960 to 1980. Comparing Figure 1 and Figure 3, it can be noted that there was increase in notification efficiency after the introduction of measles vaccination.

\section{Measles outbreak 2013 to 2014}

From 2013 and 2014, a total of 67,029 suspected measles cases were reported. More than half (52\%) of suspected cases were males and the highest number of suspected measles notifications came from NCR (26\%), Region 4A (16\%) and Region 3 (10\%). Children in the age groups of $<1$ year and 1-5 years reported the most number of suspected measles cases at $26 \%$ for each age group. Those who were aged 16-20 years old had the next highest number of reported suspected measles cases at $13 \%$.

Data on suspected measles cases were analyzed according to case classification: discarded / non-measles cases; confirmed measles cases; and rubella-confirmed, measles compatible, and pending cases as shown in Figure 4. A total of 25,166 (38\%) confirmed measles cases were reported to PIDSR. More than half (53\%) of suspected cases were classified as measles compatible, rubella-confirmed, and pending cases while $9 \%$ were classified as discarded/non-measles cases. Children aged 1 to 5 years old had the highest proportion of confirmed measles cases (29\%), followed by children $<1$ year of age at $27 \%$ while those aged $16-20$ years old comprised $12 \%$ of confirmed cases (Figure 5). The highest proportion of reported confirmed measles cases came from Region $4 \mathrm{~A}$ (22\%), NCR (31\%) and Region 3 (12\%).

Vaccination status of all cases during the outbreak were reported to PIDSR as having received any $\mathrm{MCV}$, number of doses received, and receipt of vaccine during special campaigns. Table 3 shows the reported vaccine status of suspected measles cases according to case classification. Thirty percent were reported to have missing or unknown data on receipt of any MCV while $98 \%$ did not have information on the number of doses received and $60 \%$ did not have information on receipt of $\mathrm{MCV}$ from special campaigns.

Among suspected measles cases reported to PIDSR, the highest proportion of cases with no evidence of vaccine were seen among children less than 1 year of age $(68 \%)$, while

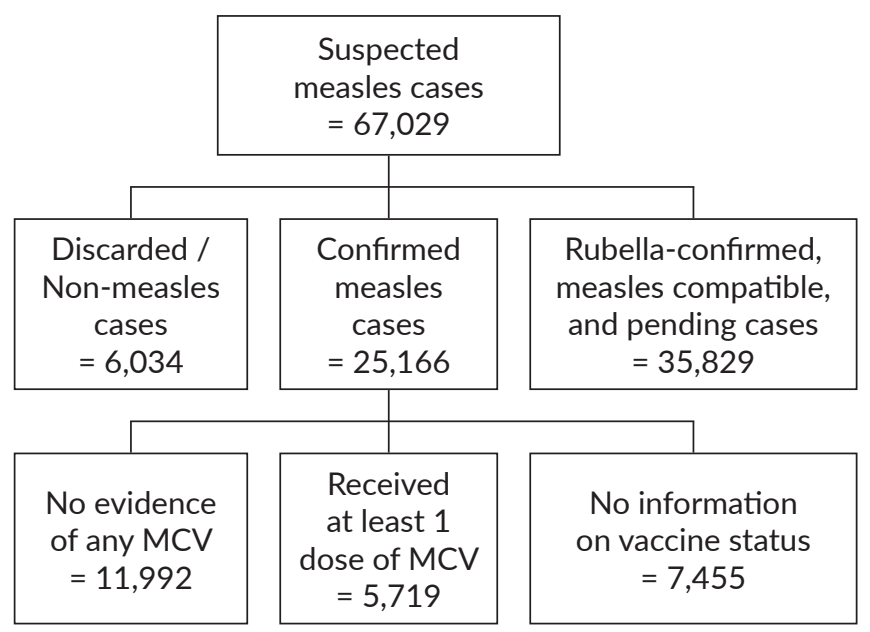

Figure 4. Classification of suspected measles cases and vaccine status of confirmed cases, Jan 2013 - 20 Dec 2014, Philippines. 


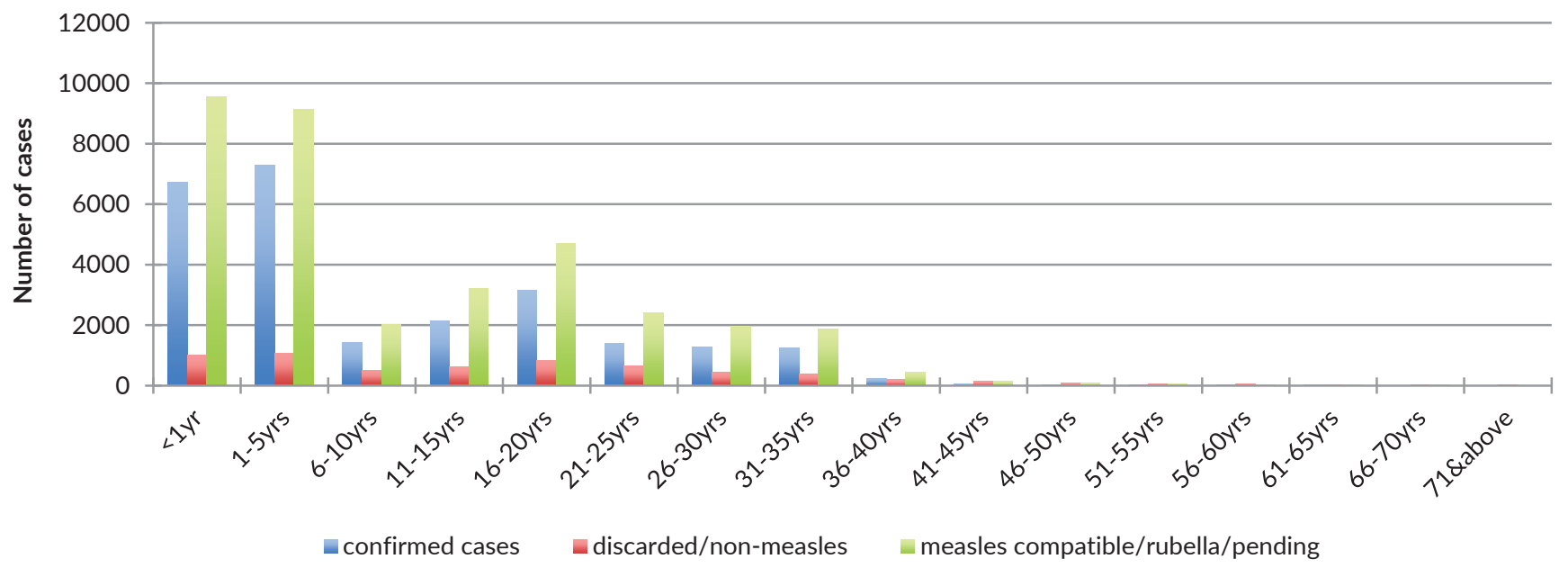

Figure 5. Number of reported confirmed, discarded and measles compatible cases by age, 2013-20 Dec 2014, Philippines.

those with evidence of receipt of vaccine were children aged 6 to 10 years (39\%) and 11 to 15 years old (39\%). There was little difference in reported vaccine status according to sex but the proportion of reported vaccination status of suspected cases varied by region. CARAGA (22\%) had the highest and ARMM (1\%) with the lowest proportion of reported cases. Incidentally, NCR (41\%) had the highest proportion of unknown vaccine status or missing information.

\section{Vaccine effectiveness}

Using the screening method, VE by birth cohort using the EPI vaccine coverage ranged from $85 \%$ to $96 \%$. The lowest VE was observed among those born from 2000 to 2001 and the highest estimates were among those born from 1990 to 1991 (Figure 6). When analyzed by age group, the lowest VE estimates were seen among those aged 26 to 30 years $(22 \%)$ while the highest estimates were noted among those aged 6 to 10 years (94\%). VE for other age groups were the following: for children aged 1 to 5 years: $90 \%$, for children aged 11 to 15 years: $66 \%$, for 16 to 20 year olds: $81 \%$, and for 21 to 25 year olds: $93 \%$.

\section{DISCUSSION}

The analyses presented in this paper indicate that the measles vaccination program had considerable impact in reducing the risk of measles in the Philippines until an outbreak occurred in 2013. The outbreak was likely a "posthoneymoon period" epidemic and not found to be related with problems in VE.

Table 3. Reported vaccine status of suspected measles cases according to case classification from January 2013 to 20 December 2014, Philippines

\begin{tabular}{|c|c|c|c|c|}
\hline & $\begin{array}{l}\text { Confirmed measles } \\
\text { frequency (\%) }\end{array}$ & $\begin{array}{l}\text { Discarded measles } \\
\text { frequency (\%) }\end{array}$ & $\begin{array}{l}\text { Rubella confirmed/measles } \\
\text { compatible frequency (\%) }\end{array}$ & Total (\%) \\
\hline \multicolumn{5}{|l|}{ Receipt of any MCV } \\
\hline No & $11,992(48)$ & $1,854(31)$ & $1,6706(47)$ & $30,552(45)$ \\
\hline Yes & $5,719(23)$ & $2,408(40)$ & $8,588(24)$ & $16,715(25)$ \\
\hline Unknown / missing & 7,455 (29) & $1,772(29)$ & $10,535(29)$ & $19,762(30)$ \\
\hline Total & $25,166(100)$ & $6,034(100)$ & $35,829(100)$ & $67,029(100)$ \\
\hline \multicolumn{5}{|l|}{ Dose } \\
\hline 0 & $40(0)$ & $51(1)$ & $6(0)$ & $97(0)$ \\
\hline 1 & 374 (1) & $573(10)$ & $211(1)$ & $1158(2)$ \\
\hline 2 & $54(1)$ & $182(3)$ & $50(0)$ & $286(0)$ \\
\hline 3 & $16(0)$ & $30(0)$ & $12(0)$ & $58(0)$ \\
\hline 4 & $1(0)$ & $2(0)$ & $0(0)$ & $3(0)$ \\
\hline Complete & $0(0)$ & $3(0)$ & $0(0)$ & $3(0)$ \\
\hline Unrecalled & $0(0)$ & $1(0)$ & $0(0)$ & $1(0)$ \\
\hline Missing & $24,681(98)$ & $5,192(86)$ & $35,550(99)$ & $65,423(98)$ \\
\hline Total & $25,166(100)$ & $6,034(100)$ & $35,829(100)$ & $67,029(100)$ \\
\hline \multicolumn{5}{|c|}{ Receipt of vaccine in special campaigns } \\
\hline No & $8,664(34)$ & $2141(35)$ & $12,010(34)$ & $22,815(34)$ \\
\hline Yes & $1331(5)$ & $565(10)$ & $1,977(6)$ & $3,873(6)$ \\
\hline Unknown/Missing & $15,171(61)$ & $3,328(55)$ & $21,842(60)$ & $40,341(60)$ \\
\hline Total & $25,166(100)$ & $6,034(100)$ & $35,829(100)$ & $67,029(100)$ \\
\hline
\end{tabular}




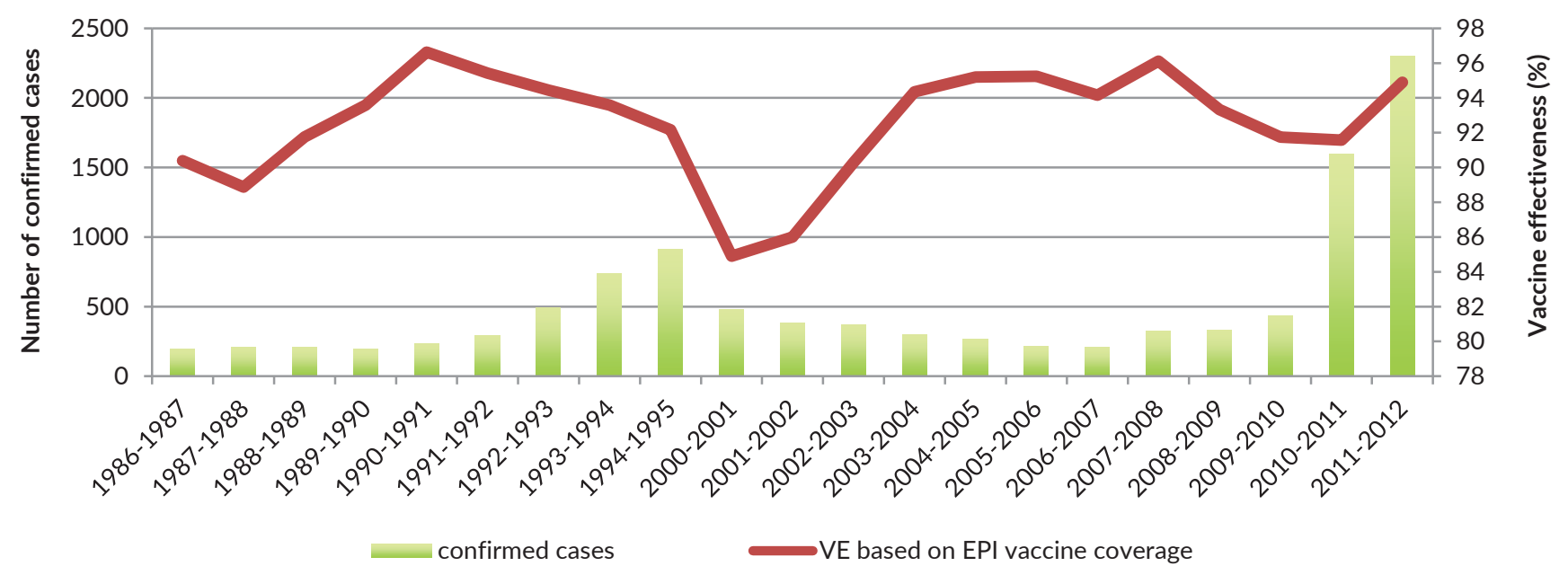

Figure 6. Estimated vaccine effectiveness on reported confirmed measles cases from January 2013 to 20 December 2014 by birth cohort.

Studies suggest that there is a threshold density of susceptible people in a defined population below which viral transmission cannot be maintained. Without vaccination, susceptible population oscillates about this threshold with an age distribution determined by the rate of loss of protection by maternal antibody and age-dependent forces of infection. With vaccination, susceptible people still oscillates about this threshold but this time determined by vaccination and the same factors mentioned earlier. The higher the vaccine coverage, the longer it will take for the susceptible pool to rebuild to its critical size. ${ }^{10}$ When the critical size has been reached, a "post honeymoon" outbreak occurs.

Live births can increase the number of susceptible people who may be infected, thus increasing the number of cases and incidence rate. A change in definition or reporting of measles cases would also have an effect on measles incidence. This paper noted a rise in the number of cases and incidence rate immediately after the introduction of MCV1 which did not signify an outbreak but likely to be due to increase in notification efficiency owing to the introduction of the measles vaccination.

It is worthwhile to note that incidence rates should be estimated using the number of cases over the population at risk. When using total population as the denominator, this will include the people who are already immune from measles and may underestimate the actual incidence rate. A cohort analysis by Fine and Clarkson used the estimated number of susceptible individuals at the beginning of each year of age of the cohort concerned as the denominator to estimate incidence rates. They found that a dramatic agespecific risk pattern is partly obscured if total population was used as denominator instead of the estimated number of susceptible. ${ }^{23}$

Notification efficiency estimates before the introduction of measles vaccine shows that estimates in the Philippines were very low. There have been limited studies on assessment of notification efficiency of measles but we assume that notification efficiency would be better in the post-vaccine period than in the pre-vaccine period. ${ }^{24}$ In England and Wales, it has been estimated that the average pre-vaccination notification efficiency for measles infections was $60 \%{ }^{22}$ This estimate was higher than notification rates in Netherlands (30\%) and Los Angeles (48\%). ${ }^{25,26}$

During the outbreak, there was a shift in age distribution among cases. The highest numbers of confirmed measles cases occurred among children aged 1 to 5 years, followed by those who were $<1$ year and then the 16 to 20 year olds. Children $<9$ months old were too young to be vaccinated since MCV1 in the Philippines were given at 9 months old. Upon breakdown of the age group $<1$ year, it was seen that $76 \%$ of children in this age group were $<9$ months of age. If the reported age was true, an implication of this would be that protection from maternal antibodies might have waned early making these children susceptible to measles. This should be interpreted with caution since one should also consider completeness and reliability of the surveillance data (i.e., correct encoding of age and birth date). In a study in Bangladesh, authors mentioned that there was a tendency to over diagnose a rash that occurs among $<3$ months as measles. ${ }^{27}$ Confirmed measles cases in the 16 to 20 years old should have received MCV1. These older cases may have missed an opportunity to be vaccinated or considered to be primary vaccine failures (those who were vaccinated but not immunized) or secondary vaccine failures (person was immunized but immunity waned).

Upon assessment of vaccination status of suspected measles cases, it was observed that 30\% had missing or unknown information on receipt of any MCV; $98 \%$ had unknown information on the number of doses; and $60 \%$ had unknown information on receipt of vaccine in special campaigns. Vaccination status was one of the most important information in surveillance data. For this dataset, it was not known how vaccination status was ascertained although the PIDSR manual specifies that vaccine status should be ascertained by checking the immunization card. 
We may assume though that the variable "receipt of any MCV" might have been obtained by recall or by checking the immunization card. The variable "number of doses" had a high proportion of unknown information since the actual immunization card would be needed to determine the number of doses and the card may not have been available at the time of recording. In special campaigns, children who received measles vaccine would be given an immunization card as proof of receiving the vaccine. However, it was uncertain if mothers kept the immunization cards given during the campaign or updated their child's immunization record in case no card was given. In some instances, the parents were not present during the actual receipt of measles vaccine during SIA and probably the mother was unaware that her child actually received measles vaccine. In Mozambique, assessment of routine surveillance data showed that only $36 \%$ of children in Maputo City and $41 \%$ of children in Manica District had information on vaccination status. The authors identified the need for quality assurance of surveillance system, motivation for clinicians to properly fill the register books, feasibility of gathering data on vaccination status of older children and training of health workers in data collection. ${ }^{28}$

Estimates of vaccine effectiveness by birth cohort and age group were obtained using the screening method. Although crude, this was a useful method for routine monitoring of VE. Estimates of VE by birth cohort ranged from $85 \%$ to $97 \%$ with vaccine coverage from $81 \%$ to $97 \%$. The lowest VE estimates were among those born from 2000 to 2001. On one hand, VE estimate by age group showed that the highest VE estimate was among those aged 6 to 10 years old at $94 \%$ while the lowest estimate was among those aged 26 to 30 years old at $23 \%$. In the screening method, the accuracy of vaccine coverage cannot be tested and analysis of risk factors for low vaccine effectiveness were not possible due to unavailability of vaccine coverage statistics stratified according to these risk factors. ${ }^{21}$ One should be wary that these estimates would be inaccurate if the proportions of population and cases vaccinated were inaccurate. ${ }^{17}$ The vaccine coverage used in computing VE was obtained from the EPI Annual Accomplishment Report of the $\mathrm{DOH}$ while the proportion of cases vaccinated were obtained from the measles outbreak dataset provided by the Epidemiology Bureau.

\section{Limitations}

The study utilized routinely collected data such as the PIDSR dataset, EPI annual report, and the routine surveillance data prior to PIDSR. Such datasets were prone to inaccurate, incomplete, or missing values. This limitation was mitigated through clarification with the agencies where data were obtained. The numbers reported in these datasets may also be an underestimate of the actual numbers of measles cases in the Philippines and the actual numbers of children vaccinated with measles vaccine due to underreporting in routine surveillance.

\section{CONCLUSION}

Reported measles incidence rates declined after the introduction of a two-dose measles vaccine. The subsequent outbreak in 2013 was not associated with problems in VE. Measles vaccination should target wider age groups that were susceptible. In deciding the target age groups, one should consider the observed shift in age distribution of measles cases seen during the outbreak. Emphasis should also be on improving measles surveillance, especially in the efficiency in reporting and completeness of measles surveillance data. Information gathered from the system can help in evidence-based decisions for a more effective plan towards measles elimination.

\section{Acknowledgments}

I would like to thank the Department of Health for sharing their measles surveillance data. I am grateful for the academic support given by my supervisors, Dr. Paul Fine and Dr. Punam Mangtani, at the London School of Hygiene and Tropical Medicine. Lastly, I would like to acknowledge my supervisor, Dr. Anna Lena Lopez, colleagues at the Institute of Child Health and Human Development, and my family and friends for the encouragement and support.

\section{Statement of Authorship}

The author has approved the final version submitted.

\section{Author Disclosure}

The author has declared no conflict of interest.

\section{Funding Source}

No external funding.

\section{REFERENCES}

1. World Health Organization. Measles. Geneva: World Health Organization; 2017 [cited Sept 2017]; Available from: http://www. who.int/mediacentre/factsheets/fs286/en/.

2. Strebel PM, Papania MJ, Fiebelkorn AP, Halsey NA. Measles. In: Plotkin S, Orenstein WA, Offit P, editors. Vaccines. 6th edition ed. Philadelphia: Elsevier Saunders; 2013.

3. Moss WJ, Griffin DE. Measles. Lancet. 2012;379(9811): 153-64. doi: 10.1016/S0140-6736(10)62352-5.

4. Durrheim DN, Crowcroft NS, Strebel PM. Measles: the epidemiology of elimination. Vaccine. 2014;32(51):6880-3. https://doi.org/10.1016/j. vaccine.2014.10.061.

5. World Health Organization. Western Pacific Region measles elimination field guide. Manila, Philippines: WHO, Western Pacific Region; 2013.

6. World Health Organization. Global Vaccine Action Plan Monitoring, Evaluation and Accountability Secretariat Annual Report 2016. Geneva: World Health Organization; 2016.

7. McFarland JW, Mansoor OD, Yang B. Accelerated measles control in the Western Pacific region. J Infect Dis. 2003;187(Suppl 1):S246-S51.

8. Takashima Y, Schluter WW, Mariano KM, et al. Progress towards measles elimination: Philippines, 1998-2014. MMWR Morbidity and Mortality Weekly Report. 2015;64(13):357-62. 
9. Sobel H, Ducusin J, De Quiroz M, Cabotaje M, Olive JM. The Philippines 2004 measles campaign: a success story towards elimination. Trop Doct. 2009;39(1):36-8.

10. McLean AR, Anderson RM. Measles in developing countries. Part II. The predicted impact of mass vaccination. Epidemiol Infect. 1988(100):419-42.

11. Chen RT, Weierbach R, Bisoffi Z, et al. A 'post-honeymoon period' measles outbreak in Muyinga sector, Burundi. Int J Epidemiol. 1994;23(1):185-93.

12. Rentsen T, Enkhtuya B, Nymadawa P, et al. Measles outbreak after a post-honeymoon period in Mongolia, 2001. Jpn J Infect Dis. 2007;60(4):198-99.

13. Antona D, Levy-Bruhl D, Baudon C, et al. Measles elimination efforts and 2008-2011 outbreak, France. Emerg Infect Dis. 2013;19(3):357-64.

14. Lopalco PL. Assessing vaccines and vaccination programmes in the field. Italian Journal of Public Health. 2009;6(3):189-93.

15. Doshi RH, Mukadi P, Shidi C, et al. Field evaluation of measles vaccine effectiveness among children in the Democratic Republic of Congo. Vaccine. 2015;33(29):3407-14.

16. Weinberg GA, Szilagyi PG. Vaccine epidemiology: efficacy, effectiveness, and the translational research roadmap. J Infect Dis. 2010;201(11):1607-10.

17. Orenstein WA, Bernier RH, Dondero TJ, et al. Field evaluation of vaccine efficacy. Bull World Health Organ. 1985;63(6):1055-68.

18. Philippine Statistics Authority. The Philippines in Figures 2015. Philippines: Philippine Statistics Authority; 2015.

19. Department of Health. The 2006 Philippine Health Statistics.Manila: Department of Health; 2006.
20. Department of Health. Manual of Procedures for the Philippine Integrated Disease Surveillance and Response. 1st Edition. Manila: Department of Health; 2008.

21. Farrington CP. Estimation of vaccine effectiveness using the screening method. Int J Epidemiol. 1993;22(4):742-46.

22. Clarkson JA, Fine PE. The efficiency of measles and pertussis notification in England and Wales. Int J Epidemiol. 1985;14(1): 153-68.

23. Fine PE, Clarkson JA. Measles in England and Wales II: The impact of the measles vaccination programme on the distribution of immunity in the population. Int J Epidemiol. 1982;11(1):15-25.

24. Davis SF, Strebel PM, Atkinson WL, et al. Reporting efficiency during a measles outbreak in New York City, 1991. Am J Public Health. 1993;83(7):1011-5.

25. Van Isterdael CED, Van Essen GA, Kuyvenhoven MM, Hoes AW, Stalman WAB, De Wit NJ. Measles incidence estimations based on the notification by general practioners were suboptimal. JClin Epidemiol. 2004;57(6):633-37.

26. Papania MJ, Strebel PM. Measles surveillance: the importance of finding the tip of the iceberg. Lancet. 2005;365(9454):100-1. DOI: 10.1016/S0140-6736(05) 17715-0.

27. Fauveau V, Chakraborty J, Sarder AM, Khan MA, Koenig MA. Measles among under-9-month-olds in rural Bangladesh: its significance for age at immunization. Bull World Health Organ. 1991; 69(1): 67-72.

28. Jani JV, Jani IV, Araújo C, Sahay S, Barreto J, Bjune G. Assessment of routine surveillance data as a tool to investigate measles outbreaks in Mozambique. BMC Infectious Diseases. 2006;6-29. https://doi. org/10.1186/1471-2334-6-29.

The Acta Medica Philippina is now accepting limited advertising for its front and back cover (colored), as well as for available spaces in some of its pages, as appropriate. For inquiries and submission of proposals, please e-mail us at actamedicaphilippina@yahoo.com 